\title{
The $B R C A 2$ gene is a potential molecular target during 5-fluorouracil therapy in human oral cancer cells
}

\author{
YOSUKE NAKAGAWA ${ }^{1,2}$, ATSUHISA KAJIHARA ${ }^{1}$, AKIHISA TAKAHASHI ${ }^{3}$, \\ NATSUKO KONDO ${ }^{2}$, EIICHIRO MORI ${ }^{4}$, TADAAKI KIRITA ${ }^{1}$ and TAKEO OHNISHI ${ }^{4}$ \\ ${ }^{1}$ Department of Oral and Maxillofacial Surgery, School of Medicine, Nara Medical University, \\ Kashihara, Nara 634-8521; ${ }^{2}$ Particle Radiation Oncology Research Center, Research Reactor Institute, \\ Kyoto University, Sennan-gun, Osaka 590-0494; ${ }^{3}$ Advanced Scientific Research Leaders Development Unit, \\ Gunma University, Maebashi, Gunma 371-8511; ${ }^{4}$ Department of Radiation Oncology, \\ School of Medicine, Nara Medical University, Kashihara, Nara 634-8521, Japan
}

Received December 19, 2013; Accepted January 28, 2014

DOI: 10.3892/or.2014.3080

\begin{abstract}
Fluorouracil (5-FU) is widely used in clinical cancer therapy. It is commonly used either alone or in combination with other drugs and/or radiation for head and neck, and other types of cancers. 5-FU induces DNA double-strand breaks (DSBs). Inhibition of the repair of 5-FU-induced DSBs may improve the therapeutic response in many tumors to this anticancer agent. The aim of the present study was to further our understanding of the pathways which are involved in the repair of 5-FU-induced DSBs. Cell survival after drug treatment was examined with colony forming assays using Chinese hamster lung fibroblast cells or Chinese hamster ovary cell lines which are deficient in DSB repair pathways involving the homologous recombination repair-related genes $B R C A 2$ and $X R C C 2$, and the non-homologous end joining repair-related genes $D N A-P K c s$ and $K u 80$. It was found that BRCA2 was involved in such repair, and may be effectively targeted to inhibit the repair of 5-FU-induced damage. Observations showed that knockdown of BRCA2 using small interference RNA suppression increased the sensitivity to 5-FU of human oral cancer cell lines (SAS and HSC3). These findings suggest that downregulation of $B R C A 2$ may be useful for sensitizing tumor cells during 5-FU chemotherapy.
\end{abstract}

\section{Introduction}

5-Fluorouracil (5-FU) is widely used as an anticancer agent. It has been commonly used either alone or in combination with otherdrugs and/or radiation for the treatment of colorectal, breast,

Correspondence to: Dr Yosuke Nakagawa, Particle Radiation Oncology Research Center, Research Reactor Institute, Kyoto University, 2 Asashiro-Nishi, Kumatori-cho, Sennan-gun, Osaka 590-0494, Japan

E-mail: y-nakagawa@rri.kyoto-u.ac.jp

Key words: 5-fluorouracil, double-strand breaks, BRCA2, oral cancer cells, DNA repair head and neck and other types of cancers (1). 5-FU belongs to the class of antimetabolite chemotherapeutics and is thought to be an inhibitor of the enzyme thymidylate synthase (TS) which plays a role in nucleotide synthesis (Fig. 1A) $(2,3)$. 5-FU is also converted to several active metabolites (Fig. 1A), including fluorouridine triphosphate (FUTP), fluorodeoxyuridine triphosphate (FdUTP) and fluorodeoxyuridine monophosphate (FdUMP). These active metabolites compromise global RNA metabolism through the incorporation of FUMP during RNA and DNA metabolism. This occurs through FdUMP-mediated inhibition of TS and incorporation of FdUMP into DNA. Inhibition of TS occurs through the formation of a ternary covalent complex consisting of TS-FdUMP-5, 10-methylenetetrahydrofolate. Once this complex is formed, cells are unable to synthesize dTMP from dUMP and cellular dUTP levels increase at the expense of dTTP. The resulting dUTP/ dTTP imbalance causes massive mis-incorporation of dUMP or FdUMP during DNA replication (4). Although DNA damage is considered to be one of the main triggers of the tumor cell killing effect of 5-FU $(5,6)$, it is not fully understood how misincorporated dUMP or FdUMP is processed and contributes to cytotoxicity. Mis-incorporated FdUMP or dUMP is recognized and excised from DNA through base excision repair (BER) or mismatch repair (MMR) (7). The repair of uracil-containing or 5-FU-containing DNA is mediated by the BER enzyme uracilDNA-glycosylase (8). However, this repair mechanism is futile in the presence of high FdUTP/dTTP ratios, and only results in additional false nucleotide incorporation. MMR plays an important role in correcting replication errors. The removal of FdUMP or dUMP by BER and MMR produces nicks and gaps in single-strand DNA (ssDNA) (5). Recently, these nicks and gaps were reported to act as triggers for the initial activation of an ATR-Chk1 signaling pathway. Chk1 molecules are activated and then stop DNA replication. During these processes, the fork of the stalled replication complex was coated with replication protein A (RPA). This event induces unstable conformations in the DNA structure. Therefore, double-strand breaks (DSBs) are subsequently induced when too many SSBs are present at stalled replication forks in 5-FU treated cells (9). 
DSBs are the most important DNA lesions which occur in cells after treatment with chemotherapy and/or radiation. The repair of these DSBs largely determines the outcome of cancer therapy. If incorrectly repaired or unrepaired themselves through the use of siRNA which targets a repair gene, DSBs may lead to cell death.

The research described here was designed to ascertain which components in DNA repair pathways significantly contribute to the repair of DSBs induced by 5-FU (Fig. 1B).

\section{Materials and methods}

Cell lines. Chinese hamster lung fibroblast cell lines were used in the present study: V79 (BRCA2 wild-type and XRCC2 wild-type); V-C8 (BRCA2-deficient), V-C8+BRCA2 (BRCA2 revertant, $\mathrm{V}-\mathrm{C} 8$ containing a $\mathrm{BAC}$ with the murine $B R C A 2$ gene) (10-12), irs1 (XRCC2-deficient), V79B (Ku80 wild-type), and XR-V15B (Ku80-deficient). Chinese hamster ovary cell lines used in the present study were CHO-K1 (DNA-PKcs wild-type) and XR-C1 (DNA-PKcs deficient). These cells were kindly provided by Drs M.Z. Zdzienicka, L.H. Thompson and A. Yasui. The human oral squamous cell carcinoma cell lines used were SAS and HSC3. Cells were obtained from the Japanese Collection of Research Bioresources (Health Science Research Resources Bank, Osaka, Japan). The cells were cultured at $37^{\circ} \mathrm{C}$ in a humidified $5 \% \mathrm{CO}_{2}$ incubator, and were grown in Dulbecco's modified Eagle's medium containing 10\% $(\mathrm{v} / \mathrm{v})$ fetal bovine serum, penicillin $(50 \mathrm{U} / \mathrm{ml})$, streptomycin $(50 \mu \mathrm{g} / \mathrm{ml})$ and kanamycin $(50 \mu \mathrm{g} / \mathrm{ml})$.

Drugs and drug treatments. 5-FU (Kyowa Hakko, Tokyo, Japan) was dissolved at a stock concentration of $100 \mathrm{mM}$ in phosphate-buffered saline (PBS). 5-FU stock solutions were stored at $-20^{\circ} \mathrm{C}$ until used. Cells were treated with medium containing 5-FU at various concentrations for $24 \mathrm{~h}$ and then rinsed twice with PBS.

Colony forming assays. Cell survival was measured using a standard colony forming assay as previously described (13). The sensitivity of each cell line was assessed by its $D_{50}$ value, i.e. from the 5-FU dose which reduced cell survival to $50 \%$. In order to accurately compare sensitivities to 5-FU in the repair defective cell lines, the relative $\mathrm{D}_{50}$ values were normalized using the $\mathrm{D}_{50}$ value of the parental cell lines.

Immunohistochemistry. Cells were grown on glass slides in $100-\mathrm{mm}$ dishes, fixed in $100 \%$ methanol (Nacalai Tesque, Inc., Kyoto, Japan) for $20 \mathrm{~min}$ at $4^{\circ} \mathrm{C}$. The cells were then permeabilized for $10 \mathrm{sec}$ at $4^{\circ} \mathrm{C}$ in $100 \%$ acetone (Nacalai Tesque) and blocked in PBS with 3\% skim milk (Nacalai Tesque) for $1 \mathrm{~h}$ at $37^{\circ} \mathrm{C}$. Cells were then incubated with anti-phospho-H2AX (Ser 139) mouse monoclonal antibody (Millipore, Billerica, MA, USA) for $1 \mathrm{~h}$ at 1:300 dilutions in PBS containing $1 \%$ BSA, and washed three times in PBS containing 1\% BSA for $10 \mathrm{~min}$. The cells were incubated with AlexaFluor 488-conjugated anti-mouse IgG secondary antibody (Invitrogen, Carlsbad, CA, USA) for $1 \mathrm{~h}$ at room temperature at 1:400 dilutions in PBS containing 1\% BSA, and washed three times for $10 \mathrm{~min}$ in PBS. Cover-glasses were mounted at 1:1,000 dilutions of 4,6-diamidino-2-phenylindole. Fluorescent images

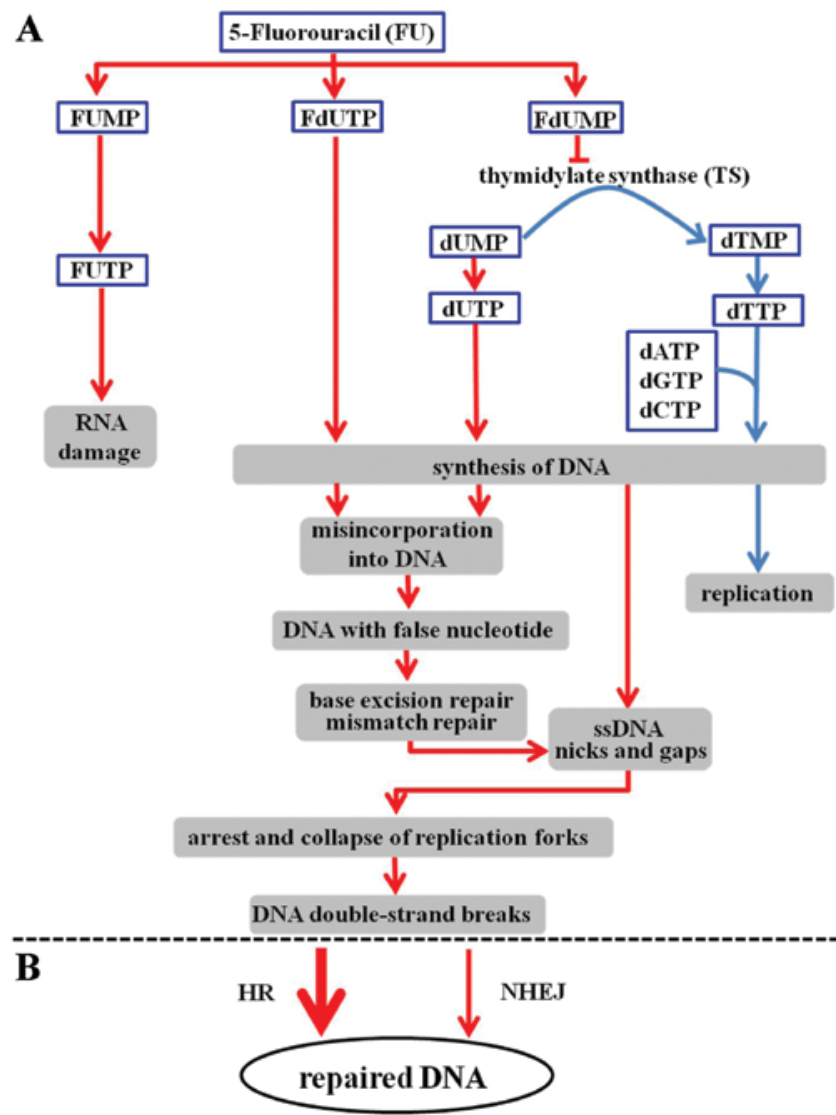

Figure 1. (A) Mechanisms of DSB induction after exposure to 5-FU. The red arrows represent the effects of 5-FU; the blue arrows represent normal reactions. HR, homologous recombination; NHEJ, non-homologous end joining. (B) DSB repair. The results of this study indicate that HR enzymes contribute largely to the repair of 5-FU-induced DSBs compared with NHEJ enzymes.

were captured for analysis using a fluorescence microscope (Keyence, Tokyo, Japan).

Flow cytometry. Cells were fixed in cold $70 \%$ methanol after a $100 \mu \mathrm{M} 5$-FU treatment for $24 \mathrm{~h}$, and maintained at $4^{\circ} \mathrm{C}$ for up to 1 week before analysis. The overall levels of phosphorylated $\mathrm{H} 2 \mathrm{AX}(\gamma \mathrm{H} 2 \mathrm{AX})$ were measured with flow cytometry as previously described (14).

Cell cycle analysis. After irradiation, cells were fixed with cold $70 \%$ methanol and stored at $4^{\circ} \mathrm{C}$ for 3 days before analysis. For cell cycle analysis, the cells were incubated for $30 \mathrm{~min}$ at room temperature with $1 \mathrm{mg} / \mathrm{ml} \mathrm{RNase}$ and $50 \mu \mathrm{g} / \mathrm{ml}$ propidium iodoide (PI), and were analyzed using a flow cytometer. The cell cycle distribution was assayed by determining the DNA content twice and calculating the average values.

siRNA transfection. The siRNA sequences used for human $B R C A 2$ and its non-specific negative control were AACAAC AAUUACGAACCAAACUU and UAUUCGCGCGUAUAG CGGUUU, respectively $(12,15)$. The siRNA duplexes were synthesized by Japan Bio Services Co., Ltd. (Saitama, Japan) and provided as a purified and annealed duplex. Transfections were carried out using Lipofectamine RNAiMAX in accordance with the manufacturer's instructions (Invitrogen). Briefly, cells were seeded at $5 \times 10^{4}$ cells per $10-\mathrm{cm}$ plate for 
$24 \mathrm{~h}$ without antibiotics. The siRNA was diluted in Opti-MEM I (Invitrogen) to produce a final siRNA concentration of $10 \mathrm{nM}$ in a $1 \mathrm{ml}$ final transfection volume. In a separate tube, $10 \mu \mathrm{l}$ of Lipofectamine RNAiMAX was added to $490 \mu \mathrm{l}$ of Opti-MEM I. The Lipofectamine RNAiMAX dilution was mixed with the diluted siRNA and incubated at room temperature for $15 \mathrm{~min}$. The complex was then added drop-wise onto the cells. The cells were incubated for $36 \mathrm{~h}$ before further processing. These cells were then trypsinized for colony forming assays.

Western blotting. Western blotting was carried out as previously described in detail (16). The membranes were then incubated with mouse monoclonal anti-BRCA2 antibody (Ab-4; Calbiochem, Darmstadt, Germany) or goat polyclonal anti-actin antibody (I-19; Santa Cruz Biotechnology, Santa Cruz, CA, USA) for the primary antibody for $1 \mathrm{~h}$ at room temperature. The membranes were washed with TPBS buffer three times and incubated with a secondary antibody conjugated to horseradish peroxidase for $1 \mathrm{~h}$. After washing three times, the blots were visualized by using an enhanced chemiluminescence method (GE Healthcare UK, Buckinghamshire, UK) following the manufacturer's protocol. The protein in the samples was quantified by scanning profiles using the Image J program (NIH, Bethesda, MD, USA). The relative ratio of the intensity of the two bands was used to determine BRCA2 protein expression levels, and these values were the average of three experiments using densitometry measurements following $\beta$-actin normalization with and without BRCA2-siRNA.

Statistical analysis. Data were compared statistically using the two-tailed Student's t-test.

\section{Results}

Repair genes which respond to 5-FU-induced DNA damage. In order to determine the relative contributions of homologous recombination (HR) and non-homologous end joining (NHEJ) repair pathways, cellular responses to 5-FU were examined using clonogenic survival assays after a 24-h exposure to 5-FU, using different cell lines deficient in DSB repair pathways. The sensitivity of each cell line was assessed according to its $D_{50}$ value, i.e. from the 5-FU dose which reduced cell survival to $50 \%$. Each $\mathrm{D}_{50}$ value was calculated from the cell survival data shown in Fig. 2A-D. In order to accurately compare 5-FU sensitivity in the repair defective cell lines, the relative $\mathrm{D}_{50}$ values were normalized using the $\mathrm{D}_{50}$ value of the corresponding proficient cell lines. The relative $\mathrm{D}_{50}$ values are listed sequentially in order of their increasing values (reflecting decreasing sensitivities to 5-FU) and are: BRCA2-deficient cells (17\%) $<X R C C 2$-deficient cells $(64 \%)<D N A-P K c s$-deficient cells $(79 \%)<K u 80$-deficient cells (82\%) (Fig. 2E). In summary, the relative $\mathrm{D}_{50}$ value of the $B R C A 2$-deficient cells was the lowest after treatment with 5-FU, reflecting the fact that these cells had the highest sensitivity to 5-FU.

Immunocytochemical staining of $\gamma H 2 A X$ foci. $\gamma \mathrm{H} 2 \mathrm{AX}$ immunocytochemical staining is an extremely sensitive method by which to detect DSBs, and was used to examine the presence of $\gamma \mathrm{H} 2 \mathrm{AX}$ foci induced by 5-FU. A typical image (Fig. 3A)

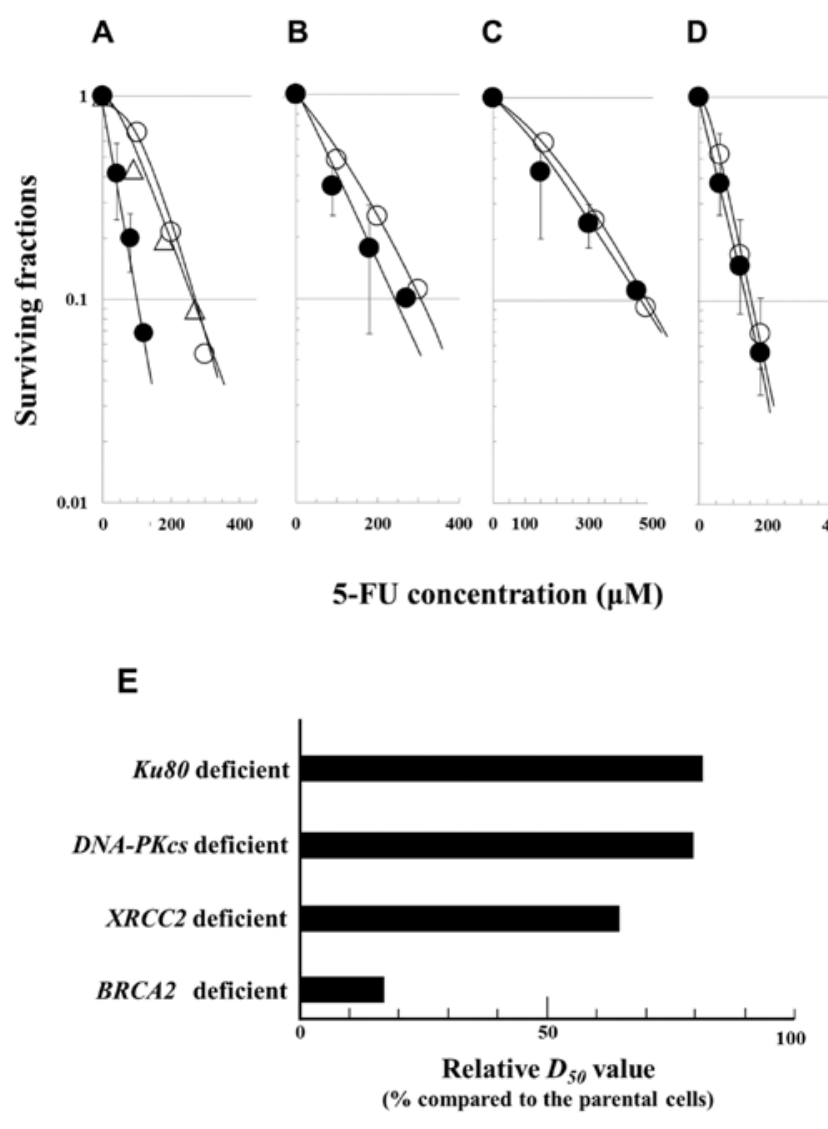

Figure 2. Contributions of HR and NHEJ. The cellular survival fractions were measured by colony forming assays following 5-FU treatment for $24 \mathrm{~h}$. (A) Closed circles, BRCA2-deficient cells; open triangles, BRCA2-revertant cells; open circles, corresponding parental cells. (B) Closed circles, XRCC2deficient cells; open circles, corresponding parental cells. (C) Closed circles, DNA-PKcs-deficient cells; open circles, corresponding parental cells. (D) Closed circles, $\mathrm{Ku} 80$-deficient cells; open circles, corresponding parental cells. (E) Relative $\mathrm{D}_{50}$ value (\% compared to the parental cells).

shows $\gamma \mathrm{H} 2 \mathrm{AX}$ foci in BRCA2-deficient cells and in the parental cells after a 24-h treatment with $100 \mu \mathrm{M} 5$-FU. In the parental cells, $\gamma \mathrm{H} 2 \mathrm{AX}$ foci in the nucleus disappeared at $16 \mathrm{~h}$ after a 5-FU treatment. In contrast, in BRCA2-deficient cells, the $\gamma \mathrm{H} 2 \mathrm{AX}$ foci in the nucleus were still present at $16 \mathrm{~h}$ after 5-FU treatment.

Phosphorylation of histone $H 2 A X$. To quantify the $\gamma \mathrm{H} 2 \mathrm{AX}-$ positive foci, the optical intensity of $\gamma \mathrm{H} 2 \mathrm{AX}$ was measured using flow cytometry. When cells were fixed with methyl alcohol immediately after the 24-h treatment with $100 \mu \mathrm{M}$ 5-FU, the intensity of $\gamma \mathrm{H} 2 \mathrm{AX}$ in the BRCA2-deficient cells was similar to that in the parental wild-type cells (Fig. 3B). After a 16-h incubation, the intensity had decreased to $25 \%$ in the parental wild-type cells, while there was almost no change in the intensity of $\gamma \mathrm{H} 2 \mathrm{AX}$ in the BRCA2-deficient cells.

Cell cycle histogram and distribution. In the untreated control cells, the fraction of the cell population in the $\mathrm{G}_{2} / \mathrm{M}$ phase in the BRCA2-deficient cells and in the parental cells was 30 and $31 \%$, respectively (Fig. $4 \mathrm{E}$ and A). Immediately following 5-FU treatment, the fraction of the population in the $\mathrm{G}_{2} / \mathrm{M}$ phase was almost the same or $\sim 25 \%$, in both cell lines. At $8 \mathrm{~h}$ following the 5-FU treatment, the $\mathrm{G}_{2} / \mathrm{M}$ phase cell fractions 

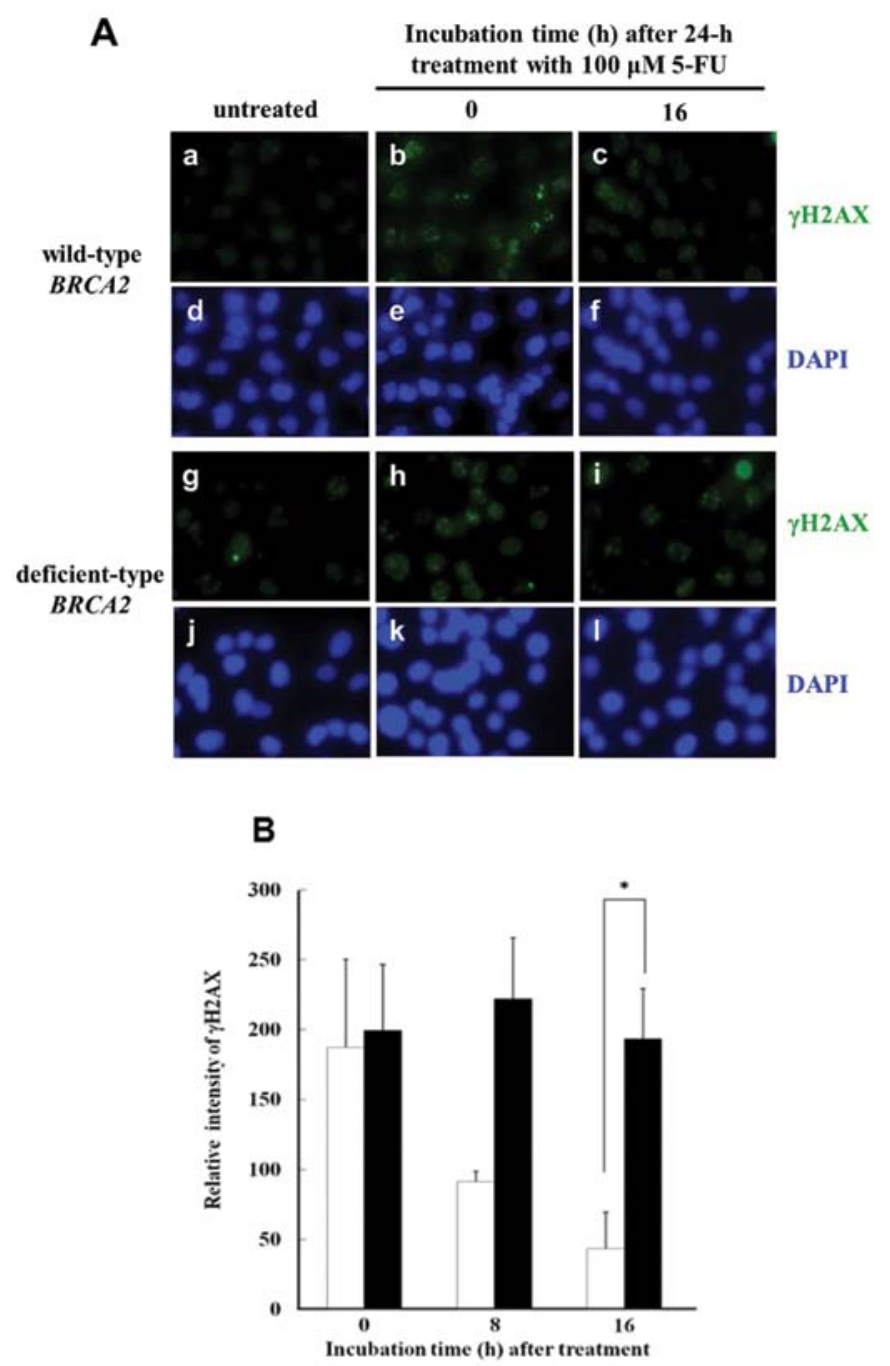

Figure 3. $\gamma \mathrm{H} 2 \mathrm{AX}$ formation following treatment with $100 \mu \mathrm{M}$ 5-FU for 24 h. (A) Typical images of $\gamma \mathrm{H} 2 \mathrm{AX}$ in BRCA2-deficient and the parental cells at the indicated time-points after a $24-\mathrm{h}$ 5-FU treatment. (a-f), wildtype BRCA2 cells; (g-l), BRCA2-deficient cells. (a-c) and (g-l), $\gamma \mathrm{H} 2 \mathrm{AX}$; (d-f) and (j-1), DAPI. (B) Induction of $\gamma \mathrm{H} 2 \mathrm{AX}$ at the indicated times following treatment with $100 \mu \mathrm{M} 5$-FU for $24 \mathrm{~h}$. Levels of $\gamma \mathrm{H} 2 \mathrm{AX}$ were measured with fluorescence intensity. Closed columns, BRCA2-deficient cells; open columns, the parental cells. ${ }^{~} \mathrm{P}<0.05$, statistically significant difference.

were between 28 and $31 \%$ (Fig. $4 \mathrm{G}$ and C) in both cell lines. At $16 \mathrm{~h}$ following the 5 -FU treatment, the $\mathrm{G}_{2} / \mathrm{M}$ phase cell fractions were 37 or $29 \%$, respectively. $\mathrm{A}_{2} / \mathrm{M}$ phase arrest was, thus, observed in the BRCA2-deficient cells (Fig. 4H), but not in the parental cells (Fig. 4D).

Effect of the silencing of BRCA2 on cellular sensitivity to $5-F U$ in human oral cancer cells. The quantity of BRCA2 protein in the BRCA2-siRNA-transfected SAS and HSC3 cells was 55 and $37 \%$, respectively, of the levels observed in the control cells transfected with the non-specific negative control siRNA (Fig. 5A and C).

To assess whether this result was pertinent to chemotherapy used against human oral cancer cells, BRCA2 expression was silenced in human oral cancer SAS and HSC3 cells using siRNA, and clonogenic survival assays were then conducted with the silenced cells. In the colony formation assays, following
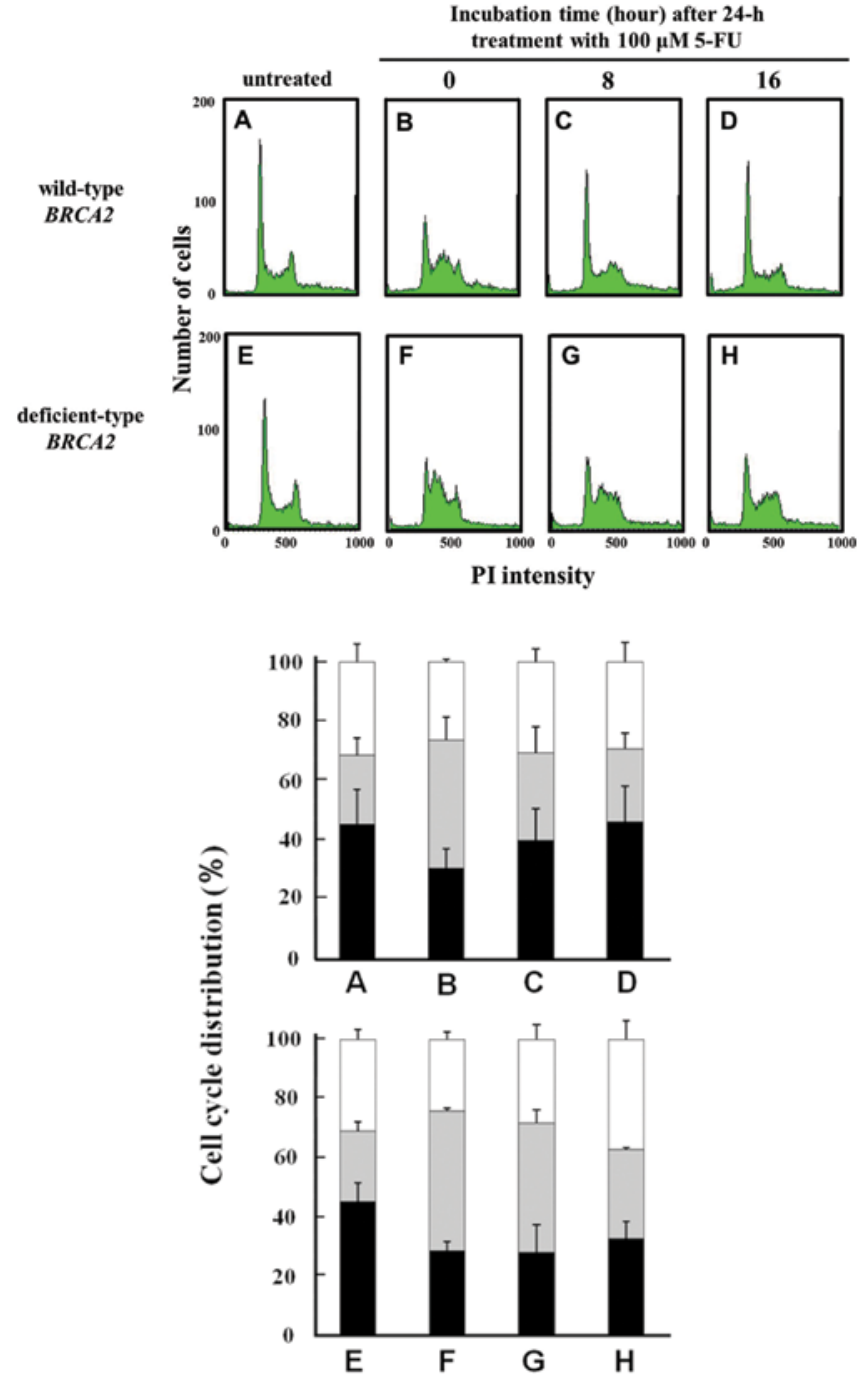

Figure 4. Cell cycle histogram and cell cycle distribution following a $100 \mu \mathrm{M}$ 5-FU treatment for $24 \mathrm{~h}$. The upper figures show cell cycle histograms obtained using flow cytometry. (A-D) Wild-type cells; (E-H) BRCA2deficient cells. (A and E) Untreated controls; (B and F) $0 \mathrm{~h}$; (C and G) $8 \mathrm{~h}$; (D and $\mathrm{H}$ ) $16 \mathrm{~h}$ following the 5-FU treatment. The lower figures show the cell cycle distributions obtained from histogram analysis. Black columns, $\mathrm{G}_{1}$; gray columns, $\mathrm{S}$; white columns, $\mathrm{G}_{2} / \mathrm{M}$.

the 5-FU treatment, BRCA2 silencing caused an approximate $25 \%$ reduction in SAS cells and an approximate $55 \%$ reduction in HSC 3 cells when compared to the cells transfected with the non-specific negative control siRNA. These results indicate that in the SAS and HSC3 cells, BRCA2 silencing increased cellular sensitivity to 5-FU (Fig. 5B and D).

\section{Discussion}

5-FU has been widely used in cancer therapy for colorectal, breast, head and neck, and other types of cancers. 5-FU belongs to the class of antimetabolite chemotherapeutics, and is thought to be an inhibitor of TS which is involved in thymidine nucleotide synthesis. Recently, several reports have described about 5-FU-induced DNA lesions and their repair in eukaryotic cells such as yeast (17) and mammalian cells (18). The repair mechanism and lesions have been studied at the molecular level, and it is possible that 5-FU induces DSBs 
A

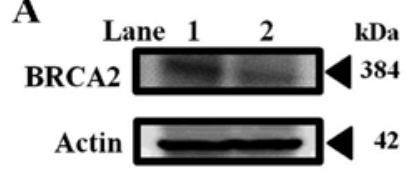

B

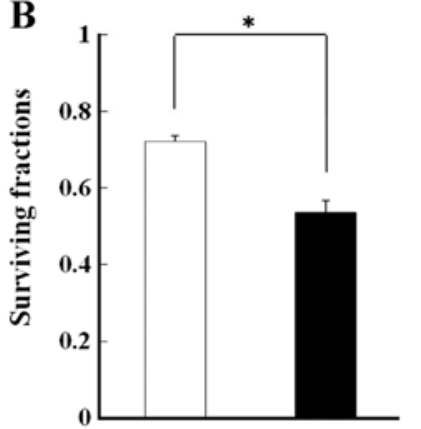

C

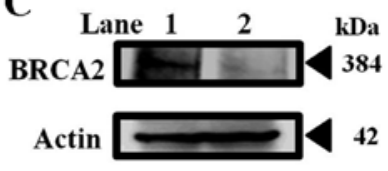

D

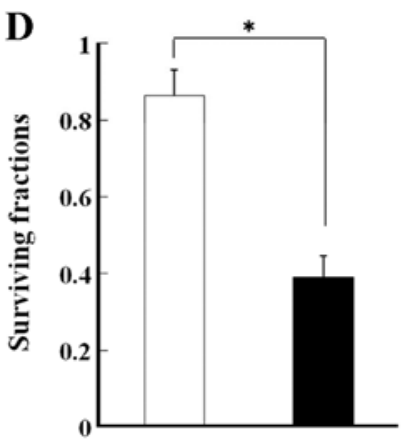

Figure 5. Effect of BRCA2-siRNA transfection in oral cancer cells. (A and B) SAS cells; (C and D) HSC3 cells. (A and C) Western blotting of BRCA2 and actin at $36 \mathrm{~h}$ following siRNA transfection. Lane 1, non-specific negative control siRNA; lane 2, BRCA2-siRNA. (B and D) Surviving fractions. The cells were transfected with siRNA and then treated with 5-FU for $24 \mathrm{~h}$. (B) $10 \mu \mathrm{M}$ 5-FU; (D) $15 \mu \mathrm{M} 5$-FU. Closed columns, BRCA2-siRNA; open columns, non-specific negative control siRNA. ${ }^{*} \mathrm{P}<0.05$, statistically significant difference.

(Fig. 1A) (9,18). When incorrect dUMP or FdUMP nucleotides are incorporated into the newly synthesized DNA strands during DNA replication, segments of ssDNA are formed from the nicks and gaps generated during the BER and MMR repair processes. These processes also result in the formation of unstable conformations in the DNA structure through the activation of the ATR-Chk1 signaling pathway, and these events can then lead to the formation of DSBs.

Quantitative analysis of DNA damage is possible by utilizing observation of $\gamma \mathrm{H} 2 \mathrm{AX}$ foci, and such measurements of $\gamma \mathrm{H} 2 \mathrm{AX}$ focus formation are extremely sensitive. $\gamma \mathrm{H} 2 \mathrm{AX}$ foci are believed to be specific indicators for the existence of DSBs induced by ionizing radiation; specifically, one $\gamma \mathrm{H} 2 \mathrm{AX}$ focus correlates with one DSB $(19,20)$. In the research described here, flow cytometry was used to quantitate relative repair activity with fluorescent measurements of $\gamma \mathrm{H} 2 \mathrm{AX}$-positive foci rather than by counting the number of positive foci per nucleus (Fig. 3A and B).

The two major DSB repair pathways are HR and NHEJ $(21,22)$. HR operates mainly by using intact sister chromatids during late $S$ and $G_{2}$ phases, but not during $G_{1}$ phase $(23,24)$. Proteins involved in HR in vertebrate cells include BRCA2, Rad52, Rad54 and Rad51 paralogs such as Rad51C-XRCC3 and Rad51B-Rad51C-Rad51D-XRCC2 (25). Rad51 activity is regulated by BRCA2 which is an upstream protein (26). Mutations in the BRCA2 gene have been frequently observed in hereditary breast (27) and ovarian cancers (28). In contrast, NHEJ is independent of cell cycle position, although its highest activity is observed in the $\mathrm{G}_{1}$ phase $(20,21)$. The main components of the NHEJ repair pathway are the DNA-PK complex (consisting of Ku70, Ku80 and DNA-PKes) and the XRCC4/ ligase IV/XLF complex (20).

An aim of the research described in the present study was to observe details in the repair pathways which repair 5-FU-induced DSBs. Cell survival was examined following 5-FU treatment using colony forming assays with Chinese hamster lung fibroblast cells, or with Chinese hamster ovary cells which are deficient in components of the NHEJ machinery (DNA-PKcs and $K u 80$ ) or in components of the HR machinery (BRCA2 and $X R C C 2$ ). The results indicated that HR enzymes, and $B R C A 2$ in particular, were responsible for a large contribution to 5-FU resistance (Figs. 1B and 2A-E). In light of this observation, attention was focused on the relationships between the BRCA2 gene and DSBs induced by 5-FU. In BRCA2 wild-type cells, the intensity of $\gamma \mathrm{H} 2 \mathrm{AX}$ foci decreased to $25 \%$ of the original value by $16 \mathrm{~h}$ following the 5 -FU treatment. In contrast, $\gamma \mathrm{H} 2 \mathrm{AX}$ focus intensity showed almost no change in the BRCA2-deficient cells. These results suggest that BRCA2 makes a major contribution to the repair of DSBs induced by 5-FU (Fig. 3A and B).

DSBs induced by 5-FU may be generated during DNA replication in the $S$ phase of the cell cycle. In addition, it was found that the cell cycle was arrested in the $G_{2} / M$ phase in the BRCA2-deficient cells (Fig. $4 \mathrm{H}$ ), but not in the parental cells (Fig. 4D). These results support to the idea that DSBs can induce $\mathrm{a}_{2} / \mathrm{M}$ phase arrest when $\mathrm{HR}$ repair does not progress.

In addition, observations showed that knockdown of the $B R C A 2$ gene by small interference RNA increased the cellular sensitivity to 5-FU in human oral cancer SAS and HSC3 cells (Fig. 5A and C). These results lead to the conclusion that disrupting BRCA2 protein synthesis may be a potentially useful strategy for improving the therapeutic efficacy of 5-FU for human oral cancer.

In summary, these observations suggest that the $B R C A 2$ gene product may serve as a molecular target for improving the efficacy of 5-FU therapy. For future therapeutic efforts, a combination of a loco-regional delivery system and the simultaneous downregulation of the BRCA2 gene may be capable of providing an effective tool to enhance the efficacy of 5-FU chemotherapy for oral cancer patients.

\section{Acknowledgements}

The present study was supported by Grants-in-Aid for Scientific Research from the Ministry of Education, Culture, Sports, Science and Technology of Japan. 


\section{References}

1. Loehrer $\mathrm{P}$, Turner S, Kubilis $\mathrm{P}$, et al: Prospective randomized trial of fluorouracil versus fluorouracil plus cisplatin in the treatment of metastatic colorectal cancer: a Hoosier Oncology Group trial. J Clin Oncol 6: 642-648, 1988

2. Welsh J, Hobbs S and Aherne G: Expression of uracil DNA glcocylase does not affect cellular sensitivity to thymidylate synthase (TS) inhibition. Eur J Cancer 39: 378-387, 2003

3. Kehler S and Ladner R: Small interfering RNA-mediated suppression of dUTPase sensitizes cancer cells to thymidylate synthase inhibition. Mol Pharmacol 66: 620-626, 2004.

4. Longley D, Harkin D and Johnston P: 5-Fluorouracil: mechanisms of action and clinical strategies. Nat Rev Cancer 3 . 330-338, 2003.

5. Waytt M and Wilson D III: Participation of DNA repair in the response to 5-fluorouracil. Cell Mol Life Sci 66: 788-799, 2009.

6. Li L, Morales J, Veigl M, et al: DNA mismatch repair (MMR)dependent 5-fluorouracil cytotoxicity and the potential for new therapeutic targets. Br J Pharmacol 158: 679-692, 2009.

7. Meyers M, Hwang A, Wagner M, et al: A role for DNA mismatch repair in sensing and responding to fluoropyrimidine damage. Oncogene 22: 7376-7388, 2003.

8. Lindahl T: An N-glycosidase from Escherichia coli that releases free uracil from DNA containing deaminated cytosine residues. Proc Natl Acad Sci USA 71: 3649-3653, 1974.

9. Fujinaka Y, Matsuoka K, Iimori M, et al: ATR-Chk1 signaling pathway and homologous recombinational repair protect cells from 5-fluorouracil cytotoxicity. DNA Repair 11: 247-258, 2012.

10. Kraakman M, Overkamp W, Lange R, et al: Brca2 (XRCC11) deficiency results in radioresistant DNA synthesis and a higher frequency of spontaneous deletions. Mol Cell Biol 22: 669-679, 2002.

11. Wiegant W, Overmeer R, Godthelp B, van Buul $\mathrm{P}$ and Zdzienicka M: Chinese hamster cell mutant, V-C8, a model for analysis of Brca2 function. Mutat Res 600: 79-88, 2006.

12. Kondo N, Takahashi A, Mori E, et al: FANCD1/BRCA2 plays predominant role in the repair of DNA damage induced by ACNU or TMZ. PLoS One 6: e19659, 2011.

13. Kondo N, Takahashi A, Mori E, et al: DNA ligase IV as a new molecular target for temozolomide. Biochem Biophys Res Commun 387: 656-660, 2009.
14. Takahashi A, Matsumoto H, Nagayama K, et al: Evidence for the involvement of double-strand breaks in heat-induced cell killing. Cancer Res 64: 8839-8845, 2004.

15. Bruun D, Folias A, Akkari Y, et al: siRNA depletion of BRCA1 but not BRCA2, causes increased genome instability in Fanconi anemia cells. DNA Repair 2: 1007-1013, 2003.

16. Yamakawa N, Takahashi A, Mori E, et al: High LET radiation enhances apoptosis in mutated $p 53$ cancer cells through caspase- 9 activation. Cancer Sci 99: 1455-1460, 2008.

17. Lauren S, Pawel J, Miral D and James T: Linking uracil base excision repair and 5-fluorouracil toxicity in yeast. Nucleic Acids Res 34: 140-151, 2004.

18. Raafat A, Ekram M and Jachen D: Targeting DNA double-strand break repair: is it the right way for sensitizing cells to 5-fluorouracil? Anticancer Drugs 40: 160-170, 2010.

19. Rothkamm K and Lobrich M: Evidence for a lack of DNA double-strand break repair in human cells exposed to very low x-ray doses. Proc Natl Acad Sci USA 100: 5057-5062, 2003.

20. Takahashi A and Ohnishi T: Does $\gamma \mathrm{H} 2 \mathrm{AX}$ focus formation depend on the presence of DNA double strand breaks? Cancer Lett 229: 171-179, 2005.

21. Jackson SP: Sensing and repairing DNA double-strand breaks. Carcinogenesis 23: 687-696,2003.

22. Kanar R, Hoeijimakers JH and Van Gent DC: Molecular mechanisms of DNA double-strand break repair. Trends Cell Biol 8: 483-489, 1998.

23. Rothkamm K, Kruger I, Thompson LH and Lobrich M: Pathways of DNA double-strand break repair during the mammalian cell cycle. Mol Cell Biol 23: 5706-5715, 2003.

24. Hinz JM, Yamada NA, Salazar EP, Tebbs RS and Thompson LH Influence of double-strand-break repair pathways on radiosensitivity throughout the cell cycle in CHO cells. DNA Repair 4: 782-792, 2005

25. Thompson L and Schild D: Homologous recombinational repair of DNA ensures mammalian chromosome stability. Mut Res 477: 131-153, 2001.

26. Davies A, Masson J, McIlwraith M, Stasiak A and Stasiak A: Role of BRCA2 in control of the RAD 51 recombination and DNA repair protein. Mol Cell 7: 273-282, 2001.

27. Ottini L, Masala G, D'Amico C, et al: BRCA1 and BRCA2 mutation status and tumor characteristics in male breast cancer: a population-based study in Italy. Cancer Res 63: 342-347, 2003.

28. Schrader K, Hurlburt J, Kalloger S, et al: Germline BRCAl and $B R C A 2$ mutations in ovarian cancer: utility of a histology-based referral strategy. Obstet Gynecol 120: 235-240, 2012. 